\title{
New Method to Characterize Phase Change Materials
}

\section{Elena PALOMO DEL BARRIO, a , Jean-Luc DAUVERGNE ${ }^{2, b}$}

\author{
${ }^{1}$ Université de Bordeaux, Laboratoire TREFLE, Esplanade des Arts et Métiers, 33405 Talence, \\ France \\ ${ }^{2}$ CNRS, Laboratoire TREFLE, Esplanade des Arts et Métiers, 33405 Talence, France \\ aelena.palomo@trefle.u-bordeaux.fr, bjean-luc.dauvergne@bordeaux.ensam.fr
}

Keywords: Energy storage, PCM, Enthalpy-temperature function, inverse methods.

\begin{abstract}
A method for thermodynamic characterization of shape-stabilized PCM based on onesingle sample and one-single experiment has been proposed. The simplicity of the experimental device is comparable to that of the T-History method. However, instead of simple energy balances as in T-History method, a numerical heat transfer model is used to retrieve the whole set of parameters/functions characterizing the PCM from temperature measurements at one-single point within the PCM. An efficient inversion technique has been proposed for that. Its most striking feature is that it allows non-parametric identification of the enthalpy-temperature function in an easy way. Such a function is retrieved by solving a problem of moving sources estimation by inversion of a linear heat conduction model.
\end{abstract}

\section{INTRODUCTION}

Phase change materials (PCM) could play an important role for an effective and economic use of thermal energy in the industrial sector, as well as in power generation based on new conversion techniques and renewable energy resources. Moreover, PCM integration in lightweight buildings is expected to be a useful way to smooth indoor temperature variations and reduce overall heating or cooling demand.

Greatest asset of PCM is their capacity to store or release thermal energy over a narrow range of temperature, as well as latent heats ranging typically from 100 to $1000 \mathrm{~kJ} / \mathrm{kg}$ for PCM undergoing solid-liquid transformations. Recently, much work has been done on shape-stabilized PCM development. Such materials are generally made of an inert matrix (polymer, wood, concrete, graphite etc.) whose porosity is completely or partially filled with a solid-liquid phase change material. The inert matrix allows structural stability and retains the PCM when in liquid state. In addition, when made of a highly conductive material, it serves to enhance the PCM thermal conductivity.

Characterization of shape-stabilized PCM usually involves measurement of thermal conductivities and heat capacities of the solid and liquid phases, as well as transition temperatures and latent heat. Moreover, for PCM undergoing melting over a range of temperature, enthalpytemperature function is required too. Characterization is thus carried out using different samples and experimental devices. For instance, thermal conductivity and thermal diffusivity can be measured, respectively, by i.e. the hot plate method and the flash method. Dynamic hot probes methods allow simultaneous determination of thermal conductivity and capacity. As for specific heat and latent heat, separate and specific DSC (Differential Scanning Calorimetric) tests are usually used. Transition temperatures are better determined using DTA (Differential Thermal Analysis) methods and enthalpy-temperature function estimation requires DSC tests in isothermal step mode [1,2].

Of the number of apparatus/tests required for complete characterization, one notices that the tests based on DTA/DSC devices generally require very small samples (some few millilitres), so that they become inappropriate for testing heterogeneous materials with large-size representative volumes. Such a problem could be partially overcome using the T-History method [3, 4], a cheap and easy 
way for the determination of latent heats and specific heats. Unfortunately, T-History method is unable to reliable estimation of transition temperatures and enthalpy-temperature functions $[5,6]$.

We propose in this paper a new method for characterization of PCM based on one-single sample and one-single experimental device. The simplicity of the experimental device is comparable to that of the T-History method: a cylinder of PCM which is heated/cooled in a furnace following specific temperature patterns (steps, isotherms and ramps). Instead of simple energy balances as in DSC or T-History methods, a heat transfer model will be used to retrieve the whole set of parameters/functions characterizing the PCM from temperature measurements at one-single point within the PCM. A powerful inversion technique has been proposed to do that. Its most interesting feature is that it allows identification of enthalpy-temperature functions in an efficient way. They are retrieved by solving a problem of time-dependent sources estimation by inversion of a linear heat conduction model. Enthalpy-temperature functions, as well as parameters derived from (heat capacities, transition temperatures, latent heat), are thus calculated in a simple way.

Compared with DSC, the proposed method yields complete thermodynamic characterization of the PCM on one-single experiment, and it allows testing heterogeneous materials with large-size representative volumes. Moreover, testing time is significantly reduced: some few hours instead of several days as required for enthalpy-temperature function measurement when using DSC in isothermal step mode.

\section{EXPERIMENTAL DEVICE AND HEAT TRANSFER MODELING}

Experimental device. A test cylinder composed of a stainless steel tube filled with PCM will be considered in this paper. Others interesting experimental configurations have been tested in [7]. Let $R$ and $L$ denote, respectively, the inner diameter of the tube and its length. Dimensions of the test cylinder are assumed to verify $L>>R$, so that heat transfer in the length direction can be neglected. The cylinder is introduced in a furnace for heating/cooling under controlled thermal boundary conditions. The thermal response $T(r, t) \quad(0 \leq r<R)$ of the PCM at $r=0$ is recorded using a thermocouple. Another thermocouple is located at the interface between the PCM and the steel $(r=R)$. Signals $y_{m}(t) \equiv T(0, t)$ and $T_{\infty}(t) \equiv T(R, t)$ (boundary condition) will be used later for PCM characterisation.

Modeling assumptions. Main assumptions employed for PCM melting/solidification modelling are: a) the PCM can be seen as a continuous medium at the macroscopic scale; b) convective heat exchanges within the PCM are negligible; c) the thermal properties (heat capacity and thermal conductivity) of the PCM are constant within each phase but heat capacity could be discontinuous between the solid and liquid; d) phase change undergoes at constant pressure; e) for multicomponent phase change materials undergoing transformation over a range of temperatures, no segregation during solidification is also assumed. The validity of these assumptions for shapestabilized PCM has been analyzed in $[8,9,10]$.

Heat transfer equations. According to the hypothesis above, energy conservation equation can be written as:

$$
\frac{\partial H(r, t)}{\partial t}=k \frac{1}{r} \frac{\partial}{\partial r}\left(r \frac{\partial T(r, t)}{\partial r}\right), \quad \forall r \in(0, R), \forall t>0
$$

$H(r, t)$ and $T(r, t)$ are, respectively, the volumetric enthalpy and the temperature at $r$ and time $t$. Enthalpy and temperature are related by:

$$
H=\left[\rho c_{s}(1-f)+\rho c_{l} f\right] T+\rho L_{f} f .
$$


$\rho$ and $c_{x}$ represent, respectively, the density and the specific heat of the PCM in liquid $(x=l)$ or solid state $(x=s) . L_{f}$ is the PCM latent heat and $k$ represents its thermal conductivity. For PCM undergoing melting/solidification at a single temperature, the liquid fraction is given by: $f=\mathcal{H}\left(T-T_{f}\right)$, where $\mathcal{H}$ represents the Heaviside function and $T_{f}$ is the melting point. For melting/solidification taking place over a range of temperatures, DSC measurements are usually required to determine the relationship existing between the liquid fraction and the temperature.

From Eq. 1 and Eq. 2, the equation governing the thermal behaviour of the PCM can be written as follows [11]:

$$
\frac{\partial T(r, t)}{\partial t}=\alpha_{s} \frac{1}{r} \frac{\partial}{\partial r}\left(r \frac{\partial T(r, t)}{\partial r}\right)+\psi(r, t ; T) \quad 0<r<R, \forall t>0
$$

with

$$
\psi(r, t ; T)=-\frac{\Delta(\rho c)}{\rho c_{s}} \frac{\partial[f(r, t ; T) T(r, t)]}{\partial t}-\frac{\rho L_{f}}{\rho c_{s}} \frac{\partial f(r, t ; T)}{\partial t}
$$

and $\alpha_{s}=k /\left(\rho c_{s}\right), \Delta(\rho c)=\rho c_{l}-\rho c_{s}$. It can be noticed that Eq. 3 is a constant-parameters heat conduction problem with a temperature-dependent source which groups all the specific terms related to phase change phenomena. Moreover, one notices that:

$$
\frac{\partial H(r, t)}{\partial t}=\rho c_{s}\left[\frac{\partial T(r, t)}{\partial t}-\psi(r, t ; T)\right] \quad 0<r<R, \forall t>0 .
$$

According to the experimental device, PCM boundary conditions are:

$$
\left.\frac{\partial T(r, t)}{\partial r}\right|_{r=0}=0 \quad \text { and } \quad-\left.k \frac{\partial T(r, t)}{\partial r}\right|_{r=R}=h\left(T(R, t)-T_{\infty}(t)\right)
$$

where $h$ represents the inverse of the thermal resistance at the interface between the steel and the PCM. As for initial conditions, we assume: $\forall r, T(r, 0)=T_{o}$.

The spatial discretisation of Eq. 3 and Eq. 6 leads to a finite dimensional formulation of the general form:

$$
\frac{d \mathbf{T}(t)}{d t}=\mathbf{A} \mathbf{T}(t)+\mathbf{B} T_{\infty}(t)+\psi(t ; T)
$$

$\mathbf{T}(t)(n \times 1)$ is the vector of temperatures at the nodes of discretisation grid and $\boldsymbol{\psi}(t ; T) \quad(n \times 1)$ is the vector of sources. Matrix A $(n \times n)$ describes heat exchanges among nodes within the PCM and matrix $\mathbf{B}(n \times 1)$ ensures the thermal link between the PCM and its environment. Both $\mathbf{A}$ and $\mathbf{B}$ are constant matrices whose elements depend of $k, \rho c_{s}$ and $h$ values. Temperature at $r=0$ $(y(t) \equiv T(0, t))$ can be written as: $y(t)=\mathbf{C T}(t)$, where $\mathbf{C}(1 \times n)$ is an appropriate zeros/one matrix.

\section{INVERSE METHOD FOR ENTHALPY-TEMPERATURE FUNCTION ESTIMATION}

Problem statement. For $H \equiv H(T)$ estimation purposes, the temperature of the air within the furnace is controlled so that it evolves from the isotherm $T_{\text {air,min }}<T_{\text {solidus }}\left(T_{\text {air,max }}>T_{\text {liquidus }}\right)$ to the 
isotherm $T_{\text {air,max }}>T_{\text {liquidus }}\left(T_{\text {air,min }}<T_{\text {solidus }}\right)$ with a constant heating (cooling) rate $\beta$. The thermal evolution of the PCM is governed by Eq. 3 and Eq. 6. Information available is $T_{\infty}(t)$ (measured by the thermocouple located at the interface steel/PCM) and $y_{m}(t) \equiv T(0, t)$ (measured by the thermocouple located at $r=0$ within the PCM), as well as values for parameters $k$ and $h$ (previously identified/measured). The unknowns of the problem are the thermal field within the PCM, $T(r, t) \quad(0<r<R)$, and the source terms $\psi(r, t)$ which group all specific phenomena related to phase change. From Eq. 5, it can be easily demonstrated that volumetric enthalpy is:

$$
H(r, t)-H(r, 0)=\rho c_{s}\left[(T(r, t)-T(r, 0))-\int_{\tau=0}^{t} \psi(r, \tau ; T) d \tau\right]
$$

Consequently, if we are able to estimate $T(r, t)$ and $\psi(r, t) \quad(0<r<R), H(r, t)$ becomes known and the enthalpy-temperature function we are looking for results from representation of $H(r, t)$ against $T(r, t)$.

Hence, let us consider the problem of estimating the thermal field $T(r, t)$ and the source terms $\psi(r, t)$ in Eq. 3 from $y_{m}(t) \equiv T(0, t)$ measured data and known boundary conditions $T_{\infty}(t)$. This is an ill-posed inverse problem in the sense of Hadamard, because the solution may be not unique or not continuous with respect to the given data. To overcome such a difficulty a large variety of techniques have been proposed. Tikonov, Alifanov, and others from the Russian school [12, 13] proposed to cast the ill-posed inverse problem into an optimisation problem with a regularised objective function. Hence, the problem we have can be mathematically formulated as a problem of finding $\psi(r, t)$ that minimises the quadratic criterion:

$$
J=\frac{1}{2} \int_{t=0}^{t_{\text {end }}}\left[y_{m}(t)-y(t)\right]^{2} d t+\frac{\mu}{2} \int_{t=0}^{t_{\text {end }}} \int_{r=0}^{R} 2 \pi r \beta(r) \psi^{2}(r, t) d r d t
$$

where $\mu>0$ is a Tikhonov regularization parameter and $\beta(r)$ a continuous function for weighting. After spatial discretisation, Eq. 9 becomes:

$$
J=\frac{1}{2} \int_{t=0}^{t_{\text {end }}}\left[y_{m}(t)-y(t)\right]^{2} d t+\frac{1}{2} \int_{t=0}^{t_{\text {end }}} \boldsymbol{\psi}^{\prime}(t) \mathbf{R} \psi(t) d t
$$

where $\psi(t)$ is, as in Eq. 7, the vector of dimension $(n \times 1)$ which results from numerical approximation of $\psi(r, t)$. As for $\mathbf{R}(n \times n)$, it is the positive definite matrix which comes from spatial dicretisation of $\mu \pi r \beta(r)$.

Taken into account the linear nature of Eq. 3 and Eq. 6, superposition principle allows writing: $y(t)=y_{1}(t)+y_{2}(t)$, where $y_{1}(t)$ and $y_{2}(t)$ represent the output from the model (Eq. 3-6) when $\psi(r, t)=0$ and when $T_{\infty}(t)=0$ respectively. Hence, the criterion $J$ can be rewritten as a function of $e(t)=y_{m}(t)-y_{1}(t)$ by replacing $y(t)$ by $y_{2}(t)$ and $y_{m}(t)$ by $e(t)$ in Eq. 10. To avoid a cumbersome notation, $y(t) \leftarrow y_{2}(t)$ and $y_{m}(t) \leftarrow e(t)$ is assumed in the following.

Problem solution. Applying Lagrange's theory and rules from calculus of variations, it can be proven [14] that the minimum of $J$ is achieved when the following three problems are satisfied simultaneously:

(a) Direct problem: $d \mathbf{T}(t) / d t=\mathbf{A T}(t)+\mathbf{B} \boldsymbol{\psi}(t)$ 
(b) Adjoint problem: $-d \boldsymbol{\lambda}(t) / d t=\mathbf{A}^{\prime} \lambda(t)+\mathbf{C}^{\prime} \mathbf{C} \mathbf{T}(t)-\mathbf{C}^{\prime} y_{m}(t)$

(c) Stationary condition: $\psi(t)=-\mathbf{R}^{-1} \mathbf{B}^{\prime} \lambda(\mathrm{t})$

with $\mathbf{T}(0)=T_{o}$ and $\lambda\left(t_{f}\right)=\mathbf{0} . \mathbf{T}(t)(n \times 1)$ and $\lambda(t)(n \times 1)$ are respectively the PCM thermal state vector and the so called co-state vector. $\mathbf{M}^{\prime}(\mathbf{M}=\mathbf{A}, \mathbf{B}, \mathbf{C})$ is the $\mathbf{M}$ transposed matrix.

Introducing the stationary condition (Eq. 13) in the direct problem (Eq. 11) yields to the following two-point boundary-value problem:

$$
\begin{aligned}
& d \mathbf{T}(t) / d t=\mathbf{A} \mathbf{T}(t)-\mathbf{B R}^{-1} \mathbf{B}^{\prime} \lambda(t) \\
& -d \lambda(t) / d t=\mathbf{A}^{\prime} \lambda(t)+\mathbf{C}^{\prime} \mathbf{C} \mathbf{T}(t)-\mathbf{C}^{\prime} y_{m}(t)
\end{aligned} .
$$

This problem can be easily solved using the "sweep method" $[14,15]$, which assumes that $\lambda(t)$ and $\mathbf{T}(t)$ satisfy an affine relation like $\lambda(t)=\mathbf{S}_{\infty} \mathbf{T}(t)+\mathbf{v}(t)$ for some as yet unknown matrix function $\mathbf{S}_{\infty}$ and vector function $\mathbf{v}(t)$. The solution of the inverse problem is then given by [14]:

$$
\begin{aligned}
& \frac{d}{d t}\left[\begin{array}{c}
\mathbf{T}(t) \\
-\mathbf{v}(t)
\end{array}\right]=\left[\begin{array}{cc}
\left(\mathbf{A}-\mathbf{B} \mathbf{K}_{\infty}\right) & -\mathbf{B} \mathbf{R}^{-1} \mathbf{B}^{\prime} \\
\mathbf{0} & \left(\mathbf{A}-\mathbf{B} \mathbf{K}_{\infty}\right)^{\prime}
\end{array}\right]\left[\begin{array}{l}
\mathbf{T}(t) \\
\mathbf{v}(t)
\end{array}\right]+\left[\begin{array}{c}
\mathbf{0} \\
\mathbf{H}^{\prime}
\end{array}\right] y_{m}(t) \\
& \boldsymbol{\psi}(\mathrm{t})=\left[\begin{array}{ll}
-\mathbf{K}_{\infty} & \mathbf{R}^{-1} \mathbf{B}^{\prime}
\end{array}\right]\left[\begin{array}{l}
\mathbf{T}(t) \\
\mathbf{v}(t)
\end{array}\right]
\end{aligned}
$$

with $\mathbf{T}(0)=T_{o}$ and $\mathbf{v}\left(t_{f}\right)=\mathbf{0}$. The matrix $\mathbf{K}_{\infty}$ is called Kalman gain. It is given by $\mathbf{K}(t)=\mathbf{R}^{-1} \mathbf{B}^{\prime} \mathbf{S}_{\infty}$, where $\mathbf{S}_{\infty}$ is the unique solution of the Ricatti equation:

$$
\mathbf{0}=\mathbf{A}^{\prime} \mathbf{S}_{\infty}+\mathbf{S}_{\infty} \mathbf{A}-\mathbf{S}_{\infty} \mathbf{B R}^{-1} \mathbf{B}^{\prime} \mathbf{S}_{\infty}+\mathbf{C}^{\prime} \mathbf{C}
$$

One notices that the sources we are looking for are the outputs of a linear and stationary state model whose input is the temperature measured within the PCM at $r=0$. Step-by-step, solving Eq. 15 involves: a) calculation of the time-invariant matrices $\mathbf{S}_{\infty}$ and $\mathbf{K}_{\infty} ;$ b) calculation of $\mathbf{v}(t)$ and $\mathbf{T}(t)$ by time-integration of the Eq. 15; and c) calculation of $\boldsymbol{\psi}(\mathrm{t})$. Knowing $\mathbf{T}(\mathrm{t})$ (step b) and $\boldsymbol{\psi}(\mathrm{t})$ (step c), the time evolution of the enthalpy vector $\mathbf{H}(t)(n \times 1)$ can be obtained by:

$$
\mathbf{H}(t)=\rho_{s} c_{s}\left\{\mathbf{T}(t)-\int_{t=0}^{t} \boldsymbol{\psi}(\tau) d \tau\right\} .
$$

It must be noticed that the unknowns of the problem (temperatures, sources and enthalpies) are estimated for all times and positions. Consequently, the enthalpy-temperature function we are looking for results from either representation of $\mathbf{H}(t)$ against $\mathbf{T}(\mathrm{t})$ or representation of any of the elements of $\mathbf{H}(t)$, i.e. $H_{i}(t)(i=1 \cdots n)$, against the corresponding element of $\mathbf{T}(\mathrm{t}), T_{i}(t)$. Notice that successful inversions must lead to $\forall i, j \quad H_{i}\left(T_{i}\right)=H_{j}\left(T_{j}\right)$ when $T_{i}=T_{j}$.

Numerical tests. Some numerical analyses have been carried out in order to establish the limits of the inverse method proposed above and to identify the parameters that have a significant effect on the quality of the results. Model give by Eqs. 3-6 has been used for emulate experiments. $T_{\infty}(t)$ is 
assumed to be increased at constant rate $\beta$. PCM melting takes place over a range of temperature $\Delta T_{f}$. Dimensionless parameters associated to the problem are:

$$
B i=\frac{h R}{k}, \quad \text { Ste }=\frac{\rho c_{s} \Delta T}{\rho L_{f}}, \quad \gamma=\frac{\Delta(\rho c)}{\rho c_{s}}, \quad \beta^{*}=\frac{\beta R^{2}}{\alpha_{s} \Delta T}, \quad \Delta \theta_{f}=\frac{\Delta T_{f}}{\Delta T} \text { with } \Delta T=T_{\infty, \text { max }}-T_{\infty, \text { min }} .
$$

Table 1. Dimensionless parameters (columns 2 to 6), Tikonov regularization parameter (column 6) and root mean square error in the dimensionless enthalpy-temperature function.

\begin{tabular}{|l|l|l|l|l|l|l|l|}
\hline & $B i$ & Ste & $\gamma$ & $\beta^{*}$ & $\Delta \theta_{f}$ & $\mu$ & $r m s$ \\
\hline Case000 & 0.190 & 0.1976 & 0.0431 & 0.04968 & 0.0208 & $3.5 \times 10^{-12}$ & 0.1423 \\
\hline Case001 & $\mathbf{0 . 5 7 0}$ & 0.1976 & 0.0431 & 0.04968 & 0.0208 & $3.5 \times 10^{-14}$ & 0.3385 \\
\hline Case002 & $\mathbf{0 . 1 1 4}$ & 0.1976 & 0.0431 & 0.04968 & 0.0208 & $9.5 \times 10^{-12}$ & 0.0432 \\
\hline Case003 & 0.190 & $\mathbf{0 . 5 2 7 0}$ & 0.0431 & 0.04968 & 0.0208 & $3.0 \times 10^{-12}$ & 0.0453 \\
\hline Case004 & 0.190 & $\mathbf{0 . 1 2 1 6}$ & 0.0431 & 0.04968 & 0.0208 & $4.0 \times 10^{-12}$ & 0.2538 \\
\hline Case005 & 0.190 & 0.1976 & $\mathbf{0 . 0 0 0 0}$ & 0.04968 & 0.0208 & $3.0 \times 10^{-12}$ & 0.1274 \\
\hline Case006 & 0.190 & 0.1976 & $\mathbf{0 . 0 8 6 2}$ & 0.04968 & 0.0208 & $3.5 \times 10^{-12}$ & 0.1573 \\
\hline Case007 & 0.190 & 0.1976 & 0.0431 & 0.04968 & $\mathbf{0 . 0 1 0 4}$ & $1.5 \times 10^{-12}$ & 0.4248 \\
\hline Case008 & 0.190 & 0.1976 & 0.0431 & 0.04968 & $\mathbf{0 . 0 6 2 5}$ & $1.0 \times 10^{-14}$ & 0.0201 \\
\hline Case009 & 0.190 & 0.1976 & 0.0431 & $\mathbf{0 . 2 9 8 0 8}$ & 0.0208 & $2.0 \times 10^{-15}$ & 0.1968 \\
\hline Case010 & 0.190 & 0.1976 & 0.0431 & $\mathbf{0 . 0 1 6 5 6}$ & 0.0208 & $2.6 \times 10^{-12}$ & 0.1159 \\
\hline
\end{tabular}
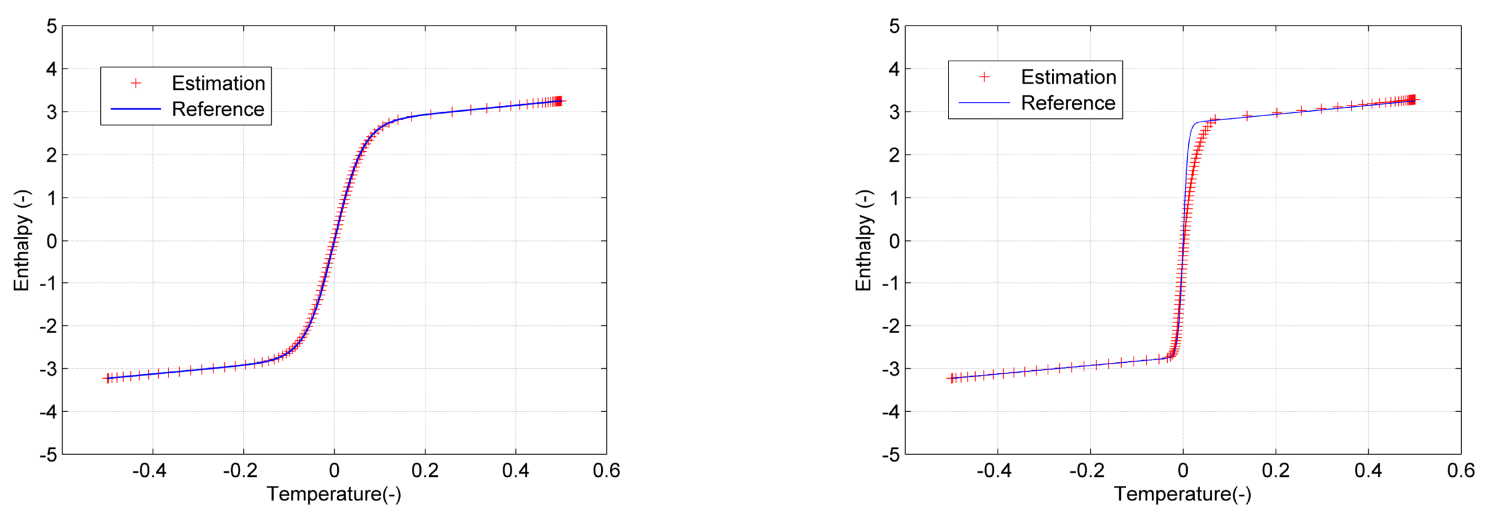

Figure 1. Estimated enthalpy-temperature function (symbols) and theoretical enthalpy-temperature function (line): Case008 (left), Case007 (right).

As shown in Table 1, different values of these parameters have been considered. For each testing case in this table, an experiment has been simulated. The algorithm described in the previous section has been applied for estimating the PCM enthalpy-temperature function from noisecorrupted $y_{m}(t)=T(0, t)$ and $T_{\infty}(\tau)$ data. The amplitude of the added noise (white noise) is $\pm 0.05^{\circ} \mathrm{C}$ (close to the noise in the experimental device). Values of the Tikhonov regularization parameter $\mu$ are provided in Table 1. The quality of the estimated enthalpy-temperature function is evaluated as the root mean squared ( $r m s$ ) of the differences between the estimated function and the theoretical one. Results achieved are reported in Table 1. It can be seen that the quality of the results strongly depends on the Biot number $(B i)$ and on the sharpness of melting $\left(\Delta T_{f}\right)$. Increasing either the Biot number or the melting sharpness leads to a degradation of the estimated enthalpy-temperature function. Biot numbers lower than $\sim 0.3$ and spread melting over a temperature interval wider than 
$0.5^{\circ} \mathrm{C}$ are recommended. Worst (case007) and best (case008) reconstructions of the enthalpytemperature function are represented in Fig. 1.

\section{EXPERIMENTAL TEST}

PCM decription. A graphite/salt composite, as those developed and studied in $[16,17,18]$, has been used in this paper for illustrating the appropriateness of our developments. It has been elaborated by uni-axial cold compression of a mixture of ENG (Expanded Natural Graphite) particles and salt powders. The salt is the binary system $\mathrm{NaNO}_{3} / \mathrm{KNO}_{3}(50 \%$ mol, laboratory graded), whit melting point at $223^{\circ} \mathrm{C}$. The sample prepared for testing is a cylinder of $76 \mathrm{~mm}$ in diameter and $200 \mathrm{~mm}$ in length. Porosity, volumetric fraction of graphite and volumetric fraction of salt are, respectively, $20 \%, 10 \%$ and $70 \%$. Graphite amount is $15 \%$ by weigth.

Experiment. The graphite/salt composite is introduced in a stainless steel tube. Top and bottom sides of the cylinder are insulated with an insulation material that can resist temperatures over 1000 ${ }^{\circ} \mathrm{C}$. The cylinder is thus introduced in a furnace for heating/cooling under controlled thermal boundary conditions. A thermocouple (type $\mathrm{K}$ ) is placed at the interface between the tube and the PCM, and another one within the PCM at $r=0$ (on the symmetry axis) and $130 \mathrm{~mm}$ from the top. Thermocouples are protected by a $1.6 \mathrm{~mm}$ inconel sheath, which is a good compromise between intrusiveness and mechanical strength.

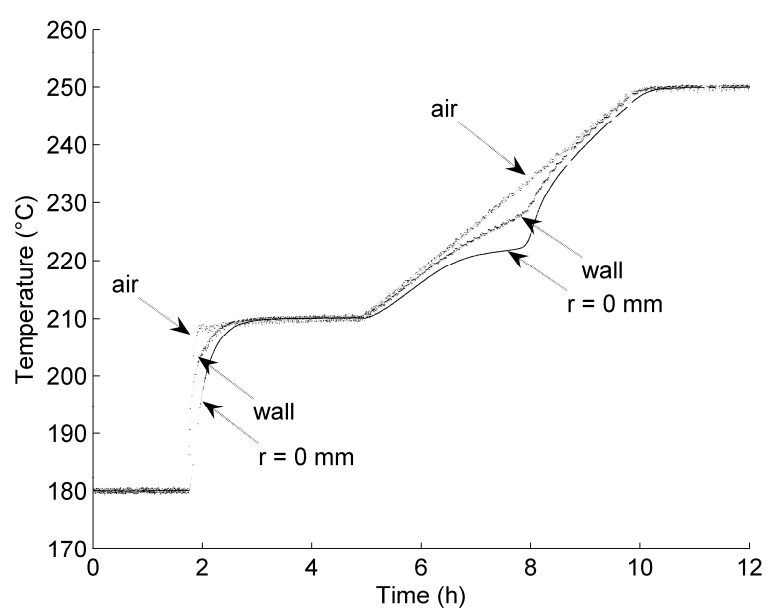

Figure 2. Data from the experiment.

During the experiment, two main periods with regard to the evolution of the temperature of the air within the furnace can be distinguished (Fig. 2, air): a) a step period where the PCM remains in solid state, which will serve for preliminary estimation of parameters $h$ and $k ; \mathrm{b}$ ) a isotherm-rampisotherm period where PCM melting takes place, which will be used for enthalpy-temperature function estimation. Wall line and $r=0 \mathrm{~mm}$ line in Fig. 2 show, respectively, the temperature measured at the interface steel/PCM and within the PCM (at $r=0$ ). To favour homogenisation of air temperature within the furnace, the fan of the furnace is continuously working.

Table 2. Graphite/salt material characterization using standard methods.

\begin{tabular}{l|c|c}
\hline & Method & Value \\
\hline Specific heat of the solid phase $\left[\mathrm{J} \cdot \mathrm{kg}^{-1} \cdot \mathrm{K}^{-1}\right]$ & DSC & 1570 \\
Specific heat of the liquid phase $\left[\mathrm{J} \cdot \mathrm{kg}^{-1} \cdot \mathrm{K}^{-1}\right]$ & DSC & 1718 \\
Latent heat $\left[\mathrm{J} \cdot \mathrm{kg}^{-1}\right]$ & DSC & 75142 \\
Melting point $\left[{ }^{\circ} \mathrm{C}\right]$ & DSC & 221.2 \\
Thermal conductivity $\left[\mathrm{W} \cdot \mathrm{m}^{-1} \cdot \mathrm{K}^{-1}\right]$ & Hot plate & 23.3 \\
Density $\left[\mathrm{kg} \cdot \mathrm{m}^{-3}\right]$ & Densimetry \& weighting & 1209 \\
\hline
\end{tabular}


At the end of the test campaign (that is after cycling), thermal and thermodynamic properties of the PCM have been measured using standard techniques. This includes: a) specific heat of the PCM at liquid and solid states; b) melting temperature and latent heat; c) radial thermal conductivity (in a plane normal to the compression axis); and d) density of the composite in solid state. Results achieved are summarized in Table 2. More detailed information can be found in [8].

Preliminary estimations. Data corresponding to the period during which the PCM stays in solid state (isotherm-step period), have been used to identify the thermal properties of the PCM and the thermal resistance associated to the interface steel/PCM. Information available is $T_{\infty}(t)$ and $y_{m}(t) \equiv T(0, t)$. The problem of $k, \rho c_{s}$ and $h$ identification is stated in a classical way. That means looking for $k, \rho c_{s}$ and $h$ values minimizing the cost function:

$$
J=\int_{t=0}^{t_{2}}\left[y_{m}(t)-y(t)\right]^{2} d t
$$

where $y(t) \equiv T(0, t)$ represents the output from model defined by Eq. 3 and Eq. 6, with $\psi(r, t)=0, \forall r, t$. The solution of this minimisation problem can be found by standard algorithms of optimisation. In this application, a Gauss-Newton algorithm has been applied. Results achieved are: $\rho c_{s}=1858354\left(J \cdot m^{-3} \cdot K^{-1}\right), \quad k=23.2\left(W \cdot m^{-1} \cdot K^{-1}\right)$ and $h=75\left(W \cdot m^{-2} \cdot K^{-1}\right)$. A high coherence is observed between heat capacity and thermal conductivity values obtained by standard measurement methods (see Table 2) and those identified by model fitting.

Estimation of the enthalpy-temperature function. The method described previously (Eqs. 1517) has been applied for estimating the enthalpy-temperature function from available data $y_{m}(t) \equiv T(0, t)$ and $T_{\infty}(t)$. Thermal parameters $k, \rho c_{s}$ and $h$ have been already identified. Tikhonov regularization parameter has been fixed at $\mu=10^{-12}$. Weighting function is $\beta(r)=1, \forall r$.
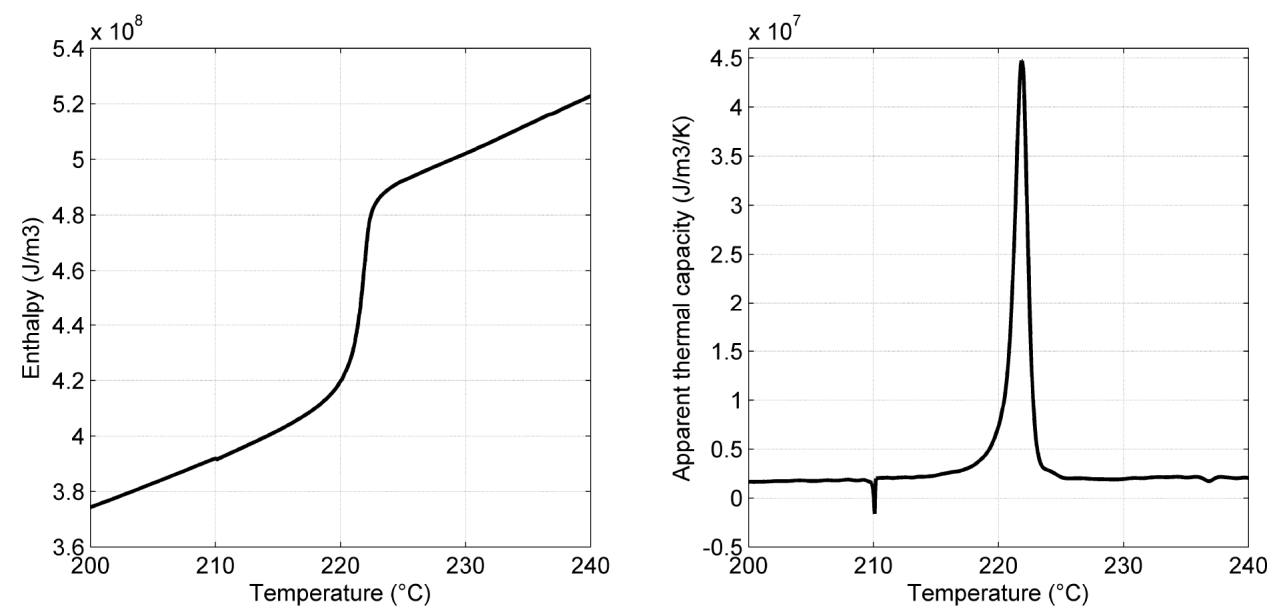

Figure 3. Enthalpy - temperature function (left) and apparent thermal capacity (right).

Figure 3 includes the estimated enthalpy-temperature function $(\hat{H}(T))$, as well as the corresponding apparent thermal capacity $(d \hat{H}(T) / d T)$. It can be seen that end of melting is observed at $223^{\circ} \mathrm{C}$, a value which is in good agreement with $\mathrm{KNO}_{3} / \mathrm{NaNO}_{3}(50 \%$ mol) melting point found in the literature (see [10] and references within). Probably due to salts impurities, a progressive as well as moderate melting is observed between 214 to $220.5^{\circ} \mathrm{C}$.

In figure 4 (left) are represented the temperature measured at $r=0$ (symbols) and the simulations performed using previously identified enthalpy-temperature function (continuous lines). The corresponding residuals (measurements - simulations) are shown in figure 4 (right). They look like 
a white noise with $\pm 0.02^{\circ} \mathrm{C}$ of amplitude. No significant bias is hence expected in the estimated enthalpy-temperature function.
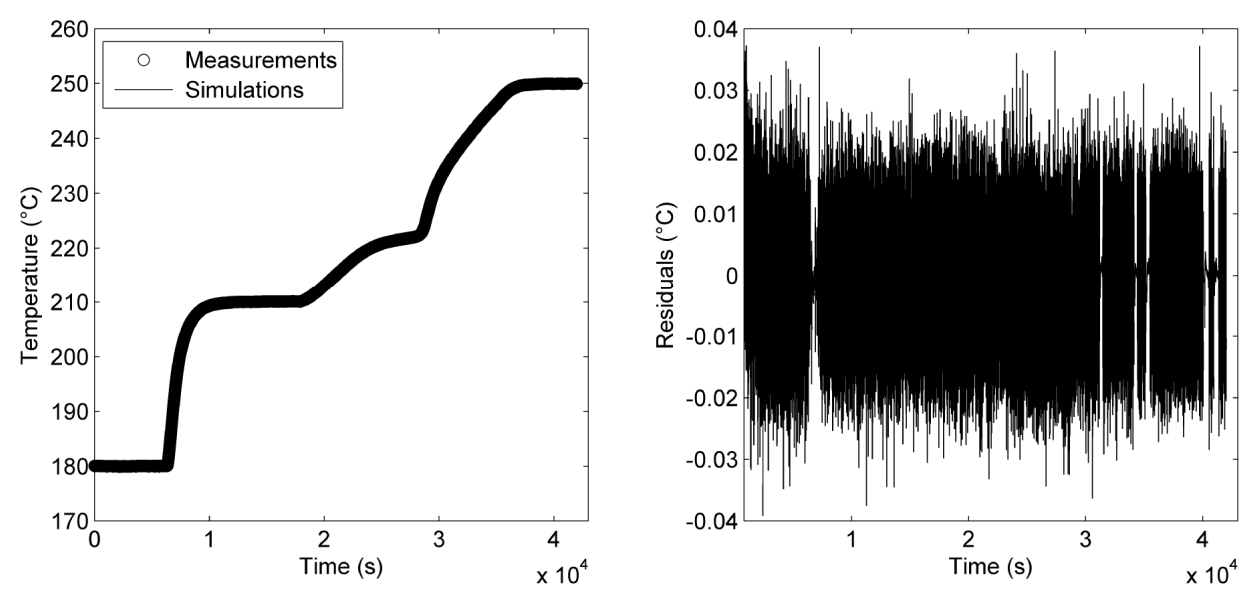

Figure 4. Measurements and simulations, on the left, and Residuals on the right.

Table 3 includes the values of the PCM thermodynamic properties obtained from the estimated enthalpy-temperature function (or alternatively from the "apparent thermal capacity - temperature" function) in figure 3. One notices they are in very good agreement with measurements in Table 2. Apparent latent heat in $\mathrm{J} / \mathrm{kg}$ has been calculated assuming apparent density in liquid state equal to $1128 \mathrm{~kg} / \mathrm{m}^{3}$. Such a value comes from specific heat in Table 2 (second line, third column) and heat capacity of the liquid phase in Table 3.

Table 3. Identified graphite/salt composite properties.

Heat capacity - solid state $\left[\mathrm{J} \cdot \mathrm{m}^{-3} \cdot \mathrm{K}^{-1}\right]$

Heat capacity - liquid state $\left[\mathrm{J} \cdot \mathrm{m}^{-3} \cdot \mathrm{K}^{-1}\right]$

Latent heat $\left[\mathrm{J}^{-\mathrm{m}^{-3}}\right]$

Latent heat $\left[\mathrm{J}^{\mathrm{kg}}{ }^{-1}\right]$

\section{PCM heating}

$1.8 \times 10^{6}$

$2.0 \times 10^{6}$

$8.127 \times 10^{8}$

72031

\section{SUMMARY}

A new method for characterization of shape-stabilized PCM based on one-single sample and one-single experimental device has been proposed. The simplicity of the experimental device is comparable to that of the T-History method: a cylinder of PCM which is heated/cooled in a furnace following specific temperature patterns (steps, isotherms and ramps). Instead of simple energy balances as in T-History method, a heat transfer model is used to retrieve the whole set of parameters/functions characterizing the PCM from temperature measurements at one-single point within the PCM. A powerful inversion technique has been proposed for that. Its main advantage is that it allows non-parametric identification of enthalpy-temperature functions. They are retrieved by solving a problem of time-dependent, moving sources estimation by inversion of a constantparameters heat conduction model. It is shown that unknown sources are the output of a linear and invariant state model whose inputs are measured temperatures within the PCM. Enthalpytemperature functions, as well as parameters derived from (heat capacities, transition temperatures, latent heat), are thus calculated in a simple way.

Numerical tests carried out show that the quality of the results achieved strongly depend on the Biot number and on the sharpness of melting. Increasing either the Biot number or the melting sharpness leads to a degradation of the estimated enthalpy-temperature function. Biot numbers lower than $\sim 0.3$ and spread melting over a temperature interval wider than $0.5^{\circ} \mathrm{C}$ are recommended. 
An experimental test for characterization of graphite/salt composites has been carried out to illustrate the appropriateness of our developments. Estimated values for PCM parameters (thermal conductivity, heat capacities, and latent heat and transition temperatures) are in good agreement with measurements coming from standard experimental techniques.

Main advantages of the proposed method with regard to DSC techniques are that it yields complete thermodynamic characterization of the PCM on one-single experiment, and that it allows testing heterogeneous materials with large-size representative volumes. Moreover, testing time is significantly reduced: some few hours instead of several days as required for enthalpy-temperature function measurement when using DSC in isothermal step mode.

\section{ACKNOWLEDGEMENTS}

The authors acknowledge the support of the European Commission for subsidizing the COSTAction TU0802, as well as ECES and SHC Programs of the International Energy Agency for subsidizing Annex 24/42.

\section{REFERENCES}

[1] M.J. Richardson: Thermochimica Acta Vol. 300 (1997), p.15.

[2] S. Rudtsch: Thermochimica Acta Vol. 382 (2002), p. 17.

[3] Y. Zhang, Y. Jiang and Y. Jiang: Meas. Sci. Technol. Vol. 10 (1999), p. 201.

[4] H. Hong, S.K. Kim and Y. Kim: Int. J. of Refrigeration Vol. 27 (2004), p. 360.

[5] A. Lázaro, E. Günther, H. Mehling, S. Hiebler, J.M. Marín and B. Zalba: Meas. Sci. Technol. Vol. 17 (2006), p. 2168.

[6] E. Günther, S. Hiebler and H. Mehling: Proc. 10th Int. Conference of Thermal Energy Storage, Ecostock 2006, New Jersey, 2006.

[7] J.L. Dauvergne: Réduction et inversion de modèles de diffusion thermique avec changement de phase, Ph.D., Université Bordeaux 1, Bordeaux, 2008.

[8] V. Morisson: Heat transfer modelling within graphite/salt composites: from the pore scale to the thermal energy storage system, Ph.D., Université Bordeaux 1, Bordeaux, 2008.

[9] X. Py, R. Olives and S. Mauran: Int. J. Heat Mass Transfer Vol. 44 (2001), p. 2727.

[10] J. Lopez: Nouveaux composites graphite/sel pour le stockage de l'énergie thermique à haute température. Etude des propriétés de changement de phase, Ph.D., Université Bordeaux 1, Bordeaux, 2007.

[11] A.N. Tikhonov and V.Y. Arsenin: Solutions of Ill-posed problems (V. H. Winston, Washington DC 1977).

[12] O.M. Alifanov: Inverse Heat Transfer Problems (Springer-Verlag, Berlin Heidelberg 1994).

[13] E. Palomo del Barrio: Inverse Problems in Science and Engineering Vol. 11 (2003), p. 515.

[14] A.E. Bryson and Y.C. Ho: Applied optimal control (Hemisphere, New York 1975).

[15] J. Lopez, J.P. Dumas and E. Palomo del Barrio: CR Mécanique Vol. 336 (2008), p. 578.

[16] J. Lopez, Z. Acem and E. Palomo del Barrio: Applied Thermal Engineering (2010), doi:10.1016/j.applthermaleng.2010.03.014.

[17] Z. Acem, J. Lopez and E. Palomo del Barrio: Applied Thermal Engineering (2010), doi:10.1016/j.applthermaleng.2010.03.013. 